\title{
Unique Indexing of Penrose Tiles and Its Application to Information-Bearing Markers
}

\author{
Hiroshi Ito \\ College of Industrial Technology \\ Nihon University \\ Narashino, Chiba Prefecture, Japan 275-8575 \\ +81-74-474-2384 \\ ito.hiroshi@nihon-u.ac.jp
}

\begin{abstract}
We propose a method of uniquely indexing tiles in a geometric pattern composed of kites and darts, known as Penrose tilings. This indexing is based on inflation, which is a property to generate larger tiles hierarchically, and independent of rotation and scaling of the pattern. By the indices of specific tiles in a pattern we can communicate information, as we do with Quick Response (QR) codes and Augmented Reality (AR) markers. We present a decoding method with these applications in mind, in which inflation is applied to identified tiles retrieving embedded code using pattern matching based on the geometric hashing. We also present a technique to identify specific tiles in an invisible way. We generated a pattern composed of 6251 tiles in which we can potentially embed 69813 bits of information using our method.
\end{abstract}

\section{CCS Concepts}

Information systems $\rightarrow$ Mobile information processing systems

\section{Keywords}

Penrose tiling; geometric pattern; inflation; deflation; information hiding; augmented reality; quick response code

\section{INTRODUCTION}

Penrose tilings have attracted many researchers and artists for their beauty and mathematical complexity [1]-[3]. Famous pieces, called darts and kites, discovered by Penrose [4] are shown in Fig.1. These pieces form prototiles tesselating a plane. Penrose tilings are non-periodic, meaning that a pattern does not match itself upon rigid shifting. This leads to the possibility that each tile is unique and discernible and is able to hold information.

Permission to make digital or hard copies of all or part of this work for personal or classroom use is granted without fee provided that copies are not made or distributed for profit or commercial advantage and that copies bear this notice and the full citation on the first page. Copyrights for components of this work owned by others than ACM must be honored. Abstracting with credit is permitted. To copy otherwise, or republish, to post on servers or to redistribute to lists, requires prior specific permission and/or a fee. Request permissions from Permissions@acm.org.

IMIP 2020, April 23-26, 2020, Tianjin, China

(C)2020 Association for Computing Machinery.

ACM ISBN 987-1-4503-7779-9/20/04 \$15.00

DOI:https://doi.org/10.1145/3399637.3399655
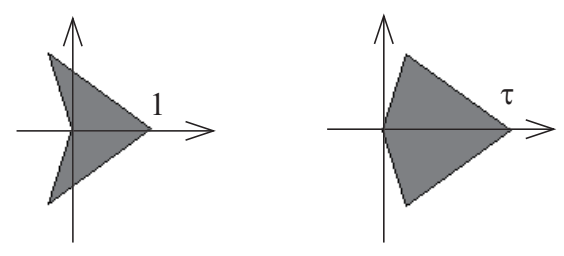

Figure 1. Prototiles known as a dart and a kite

In general, geometric patterns have been used from ancient times for decorations, architecture, and many other purposes. Letters and characters are patterns that have been functioning as information media. Calligraphy is a field of art intended to give letters and characters decorative aspects. Recently, patterns have been used as information carriers for computers. Quick response codes and data matrices are examples. Other patterns are used for augmented reality to indicate positions at which computers superimpose 3D objects in real scenery. However, most of these patterns are not visually pleasing because functionality is usually a priority [5].

We propose a method by which to associate a tile in a Penrose pattern with a number between 1 and $n$, where $n$ is the number of tiles. This association is unique, so that once a tile is identified, a number is recovered. Hence, if a pattern has a tile that is distinguishable from other tiles, which we call the base tile, the pattern can carry $\log _{2} n$ bits of information using its index directly. The pattern would then be used as an information-bearing marker, such as $\mathrm{QR}$ codes and AR markers.

In the sequel, a method of association of tiles and numbers is proposed in Section 2, followed by a description of a decoding method of information from a pattern in Section 3. In Section 4, a method by which to prevent the base tile from being noticed by the human eye is proposed. This is useful in some applications. The results obtained in the present study and further research are summarized in Section 5.

\section{TILE IDENTIFICATION}

How can we associate information with a geometric pattern? For $n$ distinct tiling shapes, $n$ integers can be as55 
shapes of Penrose tilings systematically is not straightforward. Indeed a simple inflation we use produces only two different shapes, i.e., a kite and a dart, both of larger sizes. Hence, we decided to identify each tile in a tiling, rather than depending on the shape of the contour to add information to a tiling.
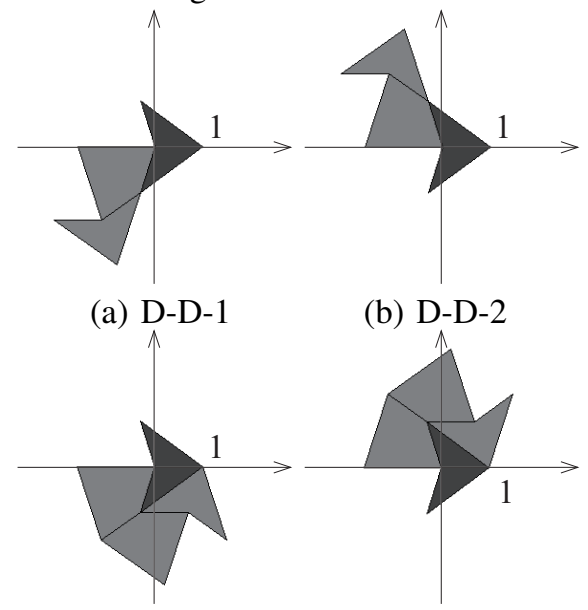

(c) D-K-1

(d) D-K-2

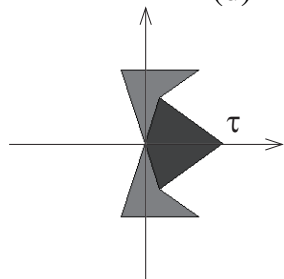

(e) K-D

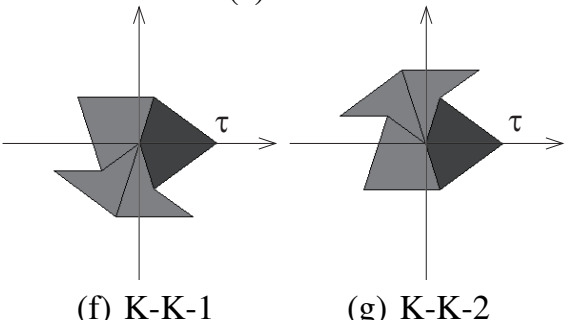

Figure 2. Seven patterns of in inflation

\subsection{Binarization}

Take $x-y$ axes for the prototile, as in Fig.1. The dart has four vertices at $(0,0),(1,0)$, and $(-(1-\tau) / 2, \pm \sin 2 \theta)$. The kite has vertices at $(0,0),(\tau, 0)$, and $(-(1-$ $\tau) / 2, \pm \sin 2 \theta)$. Here, $\tau=(1+\sqrt{5}) / 2$ and $\theta=\pi / 5$. Let the line segments $(0,0)-(1,0)$ for the dart and $(0,0)$ $(\tau, 0)$ for the kite be referred to as anchor segments. Once an anchor segment is given, the tile is fixed in space.

Penrose tilings have a fractal-like hierarchy. We can make larger kites and darts by combining small kites and darts. This is called inflation. The inverse operation is called deflation. All seven types of inflation are shown in Fig. 2, where black tiles are arranged as in Fig. 1, and gray tiles are added to black tiles, forming a scaled version of the prototile with additional half darts of the original size. For example, in Fig. 2(a), a dart is inflated to a larger dart (plus two half darts) having an anchor segment at $(-(1-\tau) / 2,-\sin 2 \theta)-(-\tau, 0)$. We consider two inflations to be different when inflated tiles have different anchors. We refer to the tile that originates the inflation as the base tile.

Now, we simply assign binary bits 0 and 1 to the dart and kite, respectively. When we inflate a tile, we assign a bit sequence of $b_{1} b_{2} b_{3}$, where $b_{1}$ and $b_{3}$ are bits assigned to the tile before and after the inflation. We need $b_{2}$ because there are at most two different inflations for the same pairs of $\left(b_{1}, b_{3}\right)$. Applying this rule, we associate 000, 010, $001,011,100,101$, and 111 with patterns (a) through (g) in Fig. 2. Note that 110 is missing because kite-to-dart inflation has only one route. Here, we refer to the bit $b_{3}$ as a joint bit. As the inflation is repeated, two bits are added to the last joint bit of the bit sequence. Fig. 3 shows a tiling obtained after eight inflations with an associated bit sequence of 11111111111111111 . This tiling has 6251 tiles.

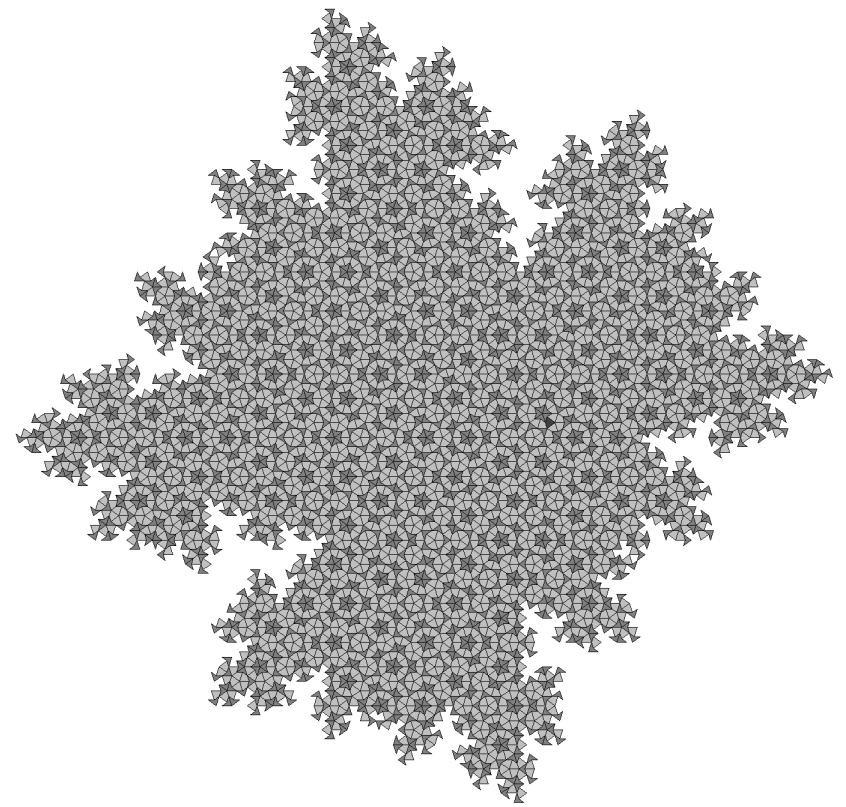

Figure 3. Pattern after 8 inflations

Every tile in a tiling is assigned at least one binary sequence. First, it is clear from Fig. 2 that this is true for a single inflation. For a tiling after $n$ inflations, take an arbitrary tile $t$ in a tiling. Identify a prototile at the $(n-1)$ th inflation that contains tile $t$. Deflate it and then identify a prototile in the smaller tiles this time at the $(n-2)$-th inflation that contains $t$, and repeat this procedure until tile $t$ itself is identified. Since inflation and deflation are inverse operations, reversing the deflation starting from $t$ up to the final tiling yields a bit sequence associated with $t$

\subsection{Indexing}

Let $m$ be the depth of inflation (which indicates how many times the inflation has been applied to the base tile), then the length of the bit sequence is $n=2 m+1$. Let $k$ be the number of tiles after inflation. Clearly we have $\log _{2} k<n$, meaning that some of the binary sequences are missing 56 
Table 1. Table for 5-bit sequences ending with 1

\begin{tabular}{c|cc} 
index & \multicolumn{2}{|c}{ code } \\
\hline 0 & 00001 & 01011 \\
1 & 00011 & \\
2 & 00101 & \\
3 & 00111 & \\
4 & 01001 & \\
5 & 01101 & \\
6 & 01111 & \\
7 & 10001 & 11001 \\
8 & 10011 & 11011 \\
9 & 10101 & \\
10 & 10111 & \\
11 & 11101 & \\
12 & 11111 &
\end{tabular}

in Section 2.1, 110 is missing in a single inflation, so all sequences having 110 at any joint bit are excluded. An example of duplicates is shown in Fig. 4, where two cases resulting in the same pattern are shown. In one case, a dart is inflated to a downward facing dart followed by inflation to an upward facing kite yielding a bit sequence of 00001. In the other case, the same dart is inflated in the other direction each time but produces the same tiling. Since each inflation is different, the inflation produces another bit sequence of 01011 .

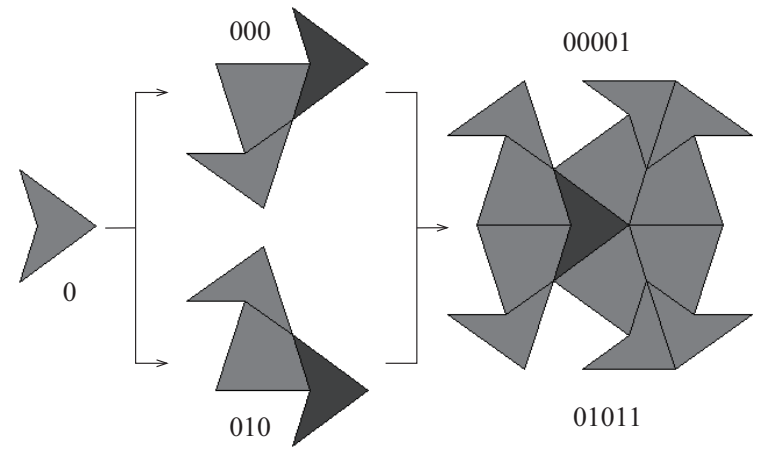

Figure 4. Example of duplicate sequences

The above discrepancy between the index and the bit sequence can be solved by introducing a conversion table listing bit sequences resulting in the same pattern and assigning these sequences the same index. For completeness, bit sequences of 110 at joint bits are also added to the table assuming that 100 and 110 are equivalents. Table 1 is an example of such a table for a bit sequence of length 5 ending with 1 . The final tiling is an inflated dart having 13 tiles. All $16\left(=2^{4}\right)$ sequences are allocated to 13 indices, which correspond to the number of tiles in the inflated pattern.

Next, we can construct an encoder and a decoder. The encoder is assigned an index, which it converts to a binary sequence using the conversion table, and generates a tiling by following each bit of the sequence. Selection of a sequence when the index has multiple entries is arbitrary. The decoder performs the opposite procedure, the details of which will be described in the next section.

\section{DECODING OF INFORMATION}

When we capture a printed pattern by camera, both geometric and signal level distortions are introduced. We do not address any preprocessing to reduce such distortions and assume that we have a registered image of a whole pattern. We also assume that the pattern has one and only one base tile. Then, our goal is to find the index associated with this tile. We do not care about the rotation of the registered image because decoding depends only on how the base tile has been inflated. For the same reason, scaling is also less relevant as long as each tile can be recognized.

\subsection{Identifying the Base Tile}

We want to first identify the base tile in a pattern. The following are the steps to do this.

1) Find contours of tiles.

2) Find corner points from the contours.

3) Pick up a pair of corner points. Apply geometric hashing with the target shape of a kite or a dart.

4) If matching is obtained, check whether the found tile is the base tile.

5) Repeat 3)-4) for all pairs of corner points.

Step 1 is straightforward if the edges are drawn by a unique color. For example, if the edges are painted black, we can extract the edges by simple thresholding. Once the contours are obtained, Step 2 identifies corner points on the contours. We compute the curvature at each point of the contour and extract points having large peak values of the curvature.

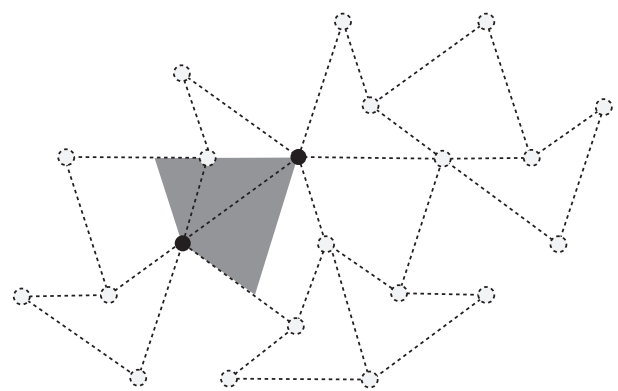

Figure 5. Matching to contours and vertices with geometric hashing

Based on the corner points, Step 3 finds base tile candidates using a technique from geometric hashing (GH) [6], which is a point-matching algorithm. Given two sets of points from model and input images, where the model shape is to be found in the input image, we compute corresponding pairs of points that minimize the mismatch of other points between two images when the images are superimposed. Let $P=\left\{\mathbf{p}_{1}, \cdots, \mathbf{p}_{M}\right\}$ and $Q=\left\{\mathbf{q}_{1}, \cdots, \mathbf{q}_{N}\right\}$ be a sets of points from input and model images. Choose a pair $\left(\mathbf{q}_{i}, \mathbf{q}_{j}\right)$ from $Q$ and compute the distortion:

$$
d_{k l}=\max _{1 \leq n \leq N} \min _{1 \leq m \leq M}\left|\mathbf{p}_{m}-T_{k l}\left(\mathbf{q}_{n}\right)\right|
$$

where $T_{k l}$ is the transform that satisfies

$$
\left(\mathbf{p}_{k}, \mathbf{p}_{l}\right)=\left(T_{k l}\left(\mathbf{q}_{i}\right), T_{k l}\left(\mathbf{q}_{j}\right)\right) .
$$


If $d_{k l}<\kappa$, where $\kappa$ is a threshold, then we found a matching with transform $T_{k l}$. In our case, $P$ is a set of points on the tile edges of the input pattern, and $Q$ is a set of points of a prototile, as defined in Fig. 1. The points $\left(\mathbf{q}_{i}, \mathbf{q}_{j}\right)$ are set to be two end points of the anchor segment, whereas $\left(\mathbf{p}_{k}, \mathbf{p}_{l}\right)$ are selected from the corner points obtained in Step 2. This only restricts the matching between the corner points of the tiles. Fig. 5 shows a case of mismatching, where two black points are selected as $\left(\mathbf{p}_{k}, \mathbf{p}_{l}\right)$. Note that some of the edges of the model do not lie on the contours.

Once a prototile matches the input image, in Step 4, we check whether we have found the base tile. If the base tile is painted a unique color, this step can be performed simply by testing whether internal pixel values are in specified ranges. In Section 4, we present a different way to identify the base tile.

\subsection{Decoding Subsequent Bits}

Subsequent bits are decoded by searching for possible inflation sequences starting from the base tile. We apply every possible inflation to the base tile and check whether the inflated pattern matches the contours of the input image. Here, we can use GH again by fixing the base tile in the input image and replacing the model with each inflated pattern from it. See Fig. 6 for an example. After identifying the black tile as the base tile and decoding the first bit as 0, two inflations for 000 and 001 are shown. Clearly 000 fits the pattern, but 001 does not. Hence, we can proceed to 000 , but not to 001 .

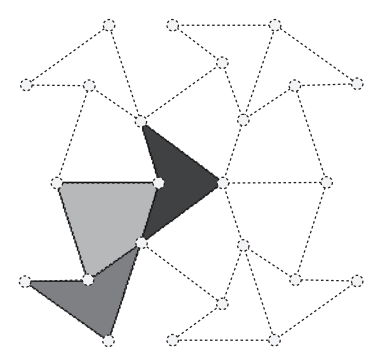

(a) 000

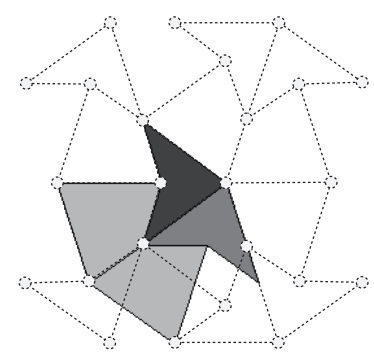

(b) 001
Figure 6. Decoding subsequent bits

Since a single inflation adds two new bits to the current bit sequence, every possible bit sequence can be represented by a path in a quaternary tree with the base tile on its root. Decoding is the process of finding possible routes on this tree. The whole tree for the above example is shown in Fig. 7. If we use the depth-first search [7] for this tree with a threshold of 0.1 for matching, we can proceed first to 000 from the root. From this node, only 00001 survives. Additional bits are not decoded because, at 00001, all four inflations do not match the pattern. Next, we go back to 001 and find that its distortion is larger than the threshold and proceed to try 010 . When the search is over, we select the longest paths, which are 00001 and 01011 , and, using Table 1 , we decode index 0 .

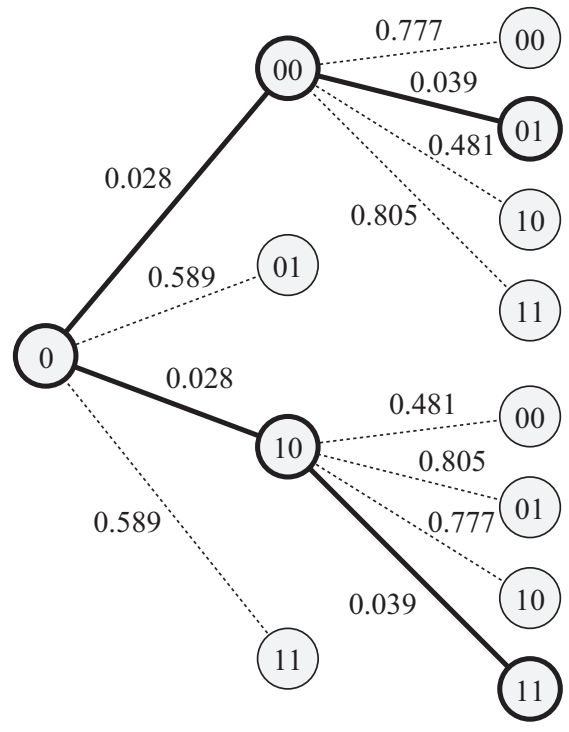

Figure 7. Decoding tree (figures on edges indicate distortions)

\section{BASE TILE CONCEALMENT}

Although identifying the base tiles is essential in decoding, it would be preferable in some applications, possibly for aesthetic reasons, where the base tile should be visually indistinguishable from other tiles. In this section we will adjust the brightness of the base tile to the same level as other tiles, while keeping the base tile detectable by computer.

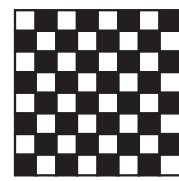

(a) texture

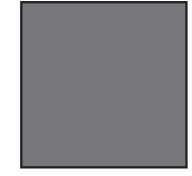

(b) solid
Figure 8. Concealment of the base tile

Let normal tiles be rendered with a solid gray indicated by signal $z(0 \leq z \leq 255)$, as in Fig. 8(b). We propose to fill the base tile with the checker flag texture, as shown in Fig. 8(a) with signals $b$ and $w$ for black and white cells, respectively. Here, the cells are rendered so small as to diffuse into uniform gray when viewed. Let $f(z)$ $(0 \leq f(z) \leq 255)$ be the function by which to convert the signal $z$ to brightness and let $g(z)=f^{-1}(z)$. Then, when we set

$$
(b, w)= \begin{cases}(0, g(2 f(z)) & \text { if } f(z)<128 \\ (g(255-2 f(z)), 255) & \text { otherwise }\end{cases}
$$

the brightness of the base and other tiles becomes the same. Hence, human eyes cannot distinguish the difference. Computers can detect the base tile by inspecting the inside texture. The above method can be applied to any color channel or combination thereof. Hence, tiles can be easily colored.

The function $g(z)$ is dependent on printers and monitors. This function is generally used to cancel the nonlinearity 58 
processes. A well-adopted form of $g(z)$ is

$$
g(z)=255\left(\frac{z}{255}\right)^{1 / \gamma}
$$

where $\gamma$ is a constant around 2.2.

\section{CONCLUSION}

We presented a method to uniquely index all of the tiles in a Penrose tiling. The proposed method provides a oneto-one correspondence between the tiles and the numbers in a systematic manner based on inflation. We presented a method by which to identify the base tile and decode the corresponding index. This decoding is assumed to be applied to rectified images after correcting projection transform, but is independent of rotation and scaling. Thus, Penrose tilings are now able to carry information by identifying the base tile and recovering the index associated with this tile. These tilings will be used as information bearing markers, such as $\mathrm{QR}$ codes and $\mathrm{AR}$ markers. We presented a method by which to make the base tile invisible to the human eye. This technique can be used optionally in applications.

In practical situations, it would be necessary to compensate distortions induced in the imaging process in order to decode information stably from distorted images. If multiple tiles were identifiable by their orders, we would be able to associate much more information, up to the number of permutations, with these tiles. If part of tilings are cropped out and the whole pattern is not available, then the proposed method would still be effective by being applied only to visible tiles. We need to investigate the adaptation of the proposed method in such cases.

\section{ACKNOWLEDGMENT}

This work was supported by a Grant-in-Aid for Scientific Research (KAKENHI) of the Japan Society for the Promotion of Science (Grant Number JP-17K00248).

\section{REFERENCES}

[1] Gardner M. 2001. The Colossal Book of Mathematics. W. W. Norton \& Company, New York, USA.

[2] Cromwell P. R. 2009. The Search for Quasi-Periodicity in Islamic 5-fold Ornament. Mathematical Intelligencer. 31, 1 (Jan. 2009), 3656.

[3] Healey, V. O. 2011. A Family of Recompositions of the Penrose Aperiodic Protoset and its Dynamic Properties. Rose-Hulman Undergraduate Mathematics Journal. 12, 2 (2011), 91-113.

[4] Penrose R. 1979. Pentaplexity: A Class of Non-Periodic Tilings of the Plane. Mathematical Intelligencer. 2, 1 (March 1979), 32-37.

[5] Garrido-Jurado S. et al. 2016. Generation of Fiducial Marker Dictionaries using Mixed Integer Linear Programming. Pattern Recognition. 51 (March 2016), 481-491.

[6] Wolfson H. J. and Rigoutsos I. 1997. Geometric Hashing: An Overview. IEEE Computational Science \& Engineering (Oct.-Dec. 1997), 10-21.

[7] Cormen T. H., Leiserson C. E., and Rivest R. L. 1997. Introduction to Algorithms. The MIT Press, Boston, USA. 